\title{
Simulation Using Initial 4D Beam Particle Distributions Synthesized from Experimental Data
}

\author{
A. Friedman, D. P. Grote; F. M. Bieniosik, C. M. Celata, \\ L. R. Prost, P. A. Seidl;
}

This article was submitted to: 2003 Particle Accelerator Conference, Portland, Oregon, USA 05/12/2003 - 05/16/2003

\section{May 1, 2003}

\section{U.S. Department of Energy}

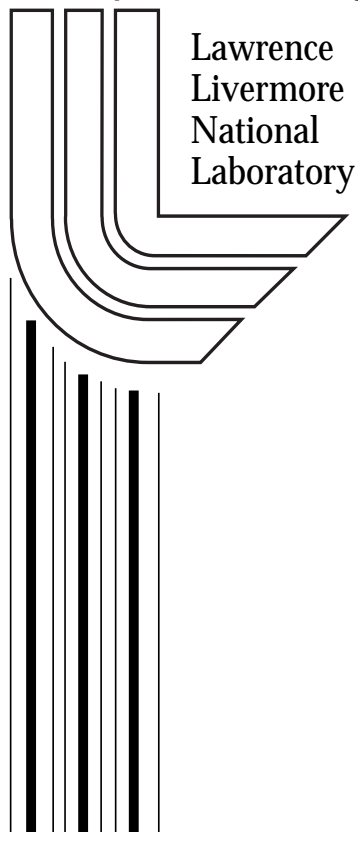




\section{DISCLAIMER}

This document was prepared as an account of work sponsored by an agency of the United States Government. Neither the United States Government nor the University of California nor any of their employees, makes any warranty, express or implied, or assumes any legal liability or responsibility for the accuracy, completeness, or usefulness of any information, apparatus, product, or process disclosed, or represents that its use would not infringe privately owned rights. Reference herein to any specific commercial product, process, or service by trade name, trademark, manufacturer, or otherwise, does not necessarily constitute or imply its endorsement, recommendation, or favoring by the United States Government or the University of California. The views and opinions of authors expressed herein do not necessarily state or reflect those of the United States Government or the University of California, and shall not be used for advertising or product endorsement purposes.

This is a preprint of a paper intended for publication in a journal or proceedings. Since changes may be made before publication, this preprint is made available with the understanding that it will not be cited or reproduced without the permission of the author.

This report has been reproduced directly from the best available copy.

Available electronically at http://www.doc.gov/bridge

Available for a processing fee to U.S. Department of Energy

And its contractors in paper from

U.S. Department of Energy

Office of Scientific and Technical Information

P.O. Box 62

Oak Ridge, TN 37831-0062

Telephone: (865) 576-8401

Facsimile: (865) 576-5728

E-mail: reports@adonis.osti.gov

Available for the sale to the public from

U.S. Department of Commerce

National Technical Information Service

5285 Port Royal Road

Springfield, VA 22161

Telephone: (800) 553-6847

Facsimile: (703) 605-6900

E-mail: orders@ntis.fedworld.gov

Online ordering: http://www.ntis.gov/ordering.htm

\section{OR}

Lawrence Livermore National Laboratory

Technical Information Department's Digital Library

http://www.llnl.gov/tid/Library.html 


\title{
SIMULATION USING INITIAL 4D BEAM PARTICLE DISTRIBUTIONS SYNTHESIZED FROM EXPERIMENTAL DATA*
}

\author{
A. Friedman, ${ }^{\dagger}$ D. P. Grote, LLNL, Livermore, CA 94550 USA \\ F. M. Bieniosek, C. M. Celata, L. R. Prost, P. A. Seidl, LBNL, Berkeley, CA 94720 USA
}

\section{Abstract}

In experiments exploring the dynamics of intense beams for Heavy Ion Fusion, detailed 2D projections of the beam's 4D transverse particle distribution function $f\left(x, y, x^{\prime} \equiv p_{x} / p_{z}, y^{\prime} \equiv p_{y} / p_{z}\right)$ are obtained at a sequence of stations, using moving slits and Faraday cups. These projections do not uniquely specify the 4D distribution, so we use maximum-entropy Monte-Carlo techniques to complete the specification and tomographically "synthesize" an approximation to $f$. Our initial studies used simulated beam data from a self-consistent 2D simulation of the High Current Experiment (HCX) [1] at LBNL. Runs initiated using a simple "semi-Gaussian" model distribution failed to exhibit an emittance evolution similar to that of the reference case. Initial distributions synthesized from the (simulated) slit scan data yielded much better agreement. We have begun to launch simulations of HCX with initial conditions synthesized from slit-scan diagnostics. We present here the techniques, initial simulation results, initial analysis of new "optical slit" data yielding projections such as $f\left(x, y, x^{\prime}\right)$, and future plans.

\section{SYNTHESIS OF 4D DISTRIBUTION FROM 2D PROJECTIONS}

We have developed algorithms which reproduce, in the limit of many particles and fine data grids, the measured distribution in the $\left(x, x^{\prime}\right)$ plane and the $\left(y, y^{\prime}\right)$ plane, as well as the data from $(x, y)$ when it is available [2]. The three-plane method we have used the most begins by assigning target "counts" $N(x, y)$ of the numbers of particles to be loaded into each spatial "bin" (area element), proportional to the measured $f$ in the bin. The sum of the $N$ 's is equal to the desired total number of simulation particles; the use of bins reduces statistical noise relative to random spatial loading following a probability distribution. We may use a sampling region which fills the entire 4-box bounded by the extremes of the measured data, or, optionally, one with "rounded corners" in the unmeasured planes. In the latter case, it is necessary to compute a corrected probability $f^{*}\left(x, x^{\prime}\right)$ by dividing the original $f\left(x, x^{\prime}\right)$ by the area in $\left(y, y^{\prime}\right)$ of the sampling region at that $\left(x, x^{\prime}\right)$. Similarly, we compute $f^{*}\left(y, y^{\prime}\right)$. The following steps are repeated until all "bin counts" have been decremented to zero: (1) Generate a random point $\left(x_{i}, y_{i}, x_{i}^{\prime}, y_{i}^{\prime}\right)$

\footnotetext{
${ }^{*}$ Work performed under the auspices of the U.S. DoE by the University of California, Lawrence Livermore and Lawrence Berkeley National Laboratories under Contract Nos. W-7405-Eng-48 and DE-AC03$76 \mathrm{SF} 00098$.

† af@1lnl.gov
}

in the 4-box; (2) Accept the point as the coordinates of a particle only if it falls within the sampling region, the bin count $N\left(x_{i}, y_{i}\right)>0, f^{*}\left(x_{i}, x_{i}^{\prime}\right)>\operatorname{Random}(0,1)$, and $f^{*}\left(y_{i}, y_{i}^{\prime}\right)>\operatorname{Random}(0,1)$; (3) If the point is accepted, decrement $N\left(x_{i}, y_{i}\right)$. Because the tomography problem is underdetermined, the sampling region is almost arbitrary and must be specified empirically. We have generally employed one [2] which consists of the intersection of the interiors of a 4-ellipsoid and four 4-cylinders, all with semiaxes of order the extent of the data along the principal axes. As is noted below, in our case it may be better to omit or modify the sampling-region constraint.

\section{SIMULATION USING MODEL DATA FROM A REFERENCE SIMULATION}

In order to to assess the potential utility of launching sinulations using experimental data, we began with a selfconsistent "transverse slice" WARP [3] simulation of HCX beginning at the source, for which the true 4D distribution was known. Input to the syntheses consisted of projectional phase-space densities (obtained by nearest-gridpoint weighting of the simulated particles from the reference run at the entrance to the HCX transport line) in the $\left(x, x^{\prime}\right)$ and $\left(y, y^{\prime}\right)$ planes, and, for the 3-plane synthesis, the $(x, y)$ plane. Projections of the input distribution are shown in Fig. 1. Some results of one such synthesis are

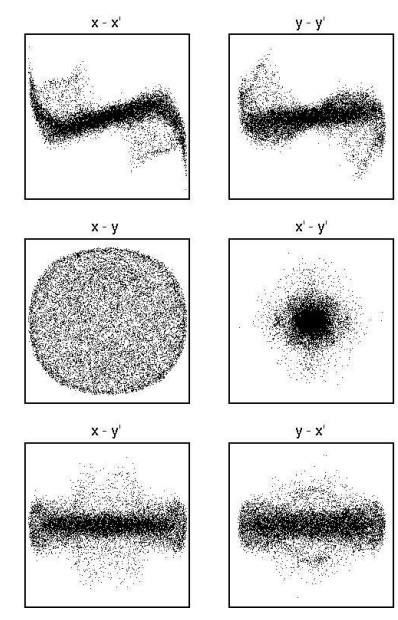

Figure 1: Projections of sampled particles onto principal planes at injector exit, for the reference simulation.

shown in Fig. 2; here the input planes $\left(x, x^{\prime}\right)$ and $\left(y, y^{\prime}\right)$ are not shown because they are faithfully reproduced, by design. Note that the synthesis fails to recreate the structures in the $\left(x, y^{\prime}\right)$ and $\left(y, x^{\prime}\right)$ planes. 

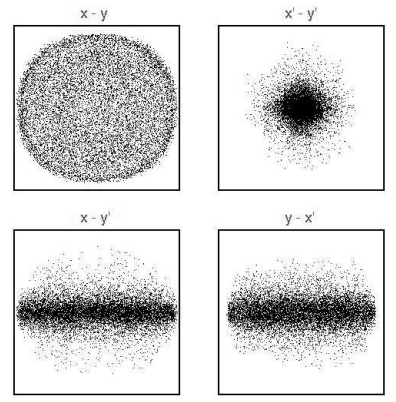

Figure 2: Projections of sampled particles onto principal planes at injector exit for 3-plane synthesized distribution.

Fig. 3 compares the emittance evolution in runs with various initial particle distributions [4]; all begin at the injector exit $(z=-3.11 \mathrm{~m})$.

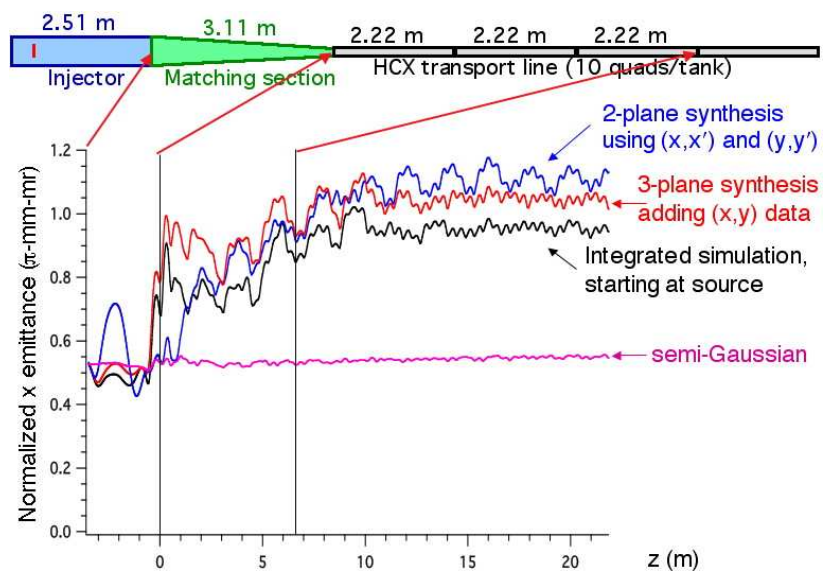

Figure 3: Simulation results: evolution of $\left(x, x^{\prime}\right)$ emittance for self-consistent, 2-plane reconstruction, 3-plane reconstruction, and semi-Gaussian beams.

\section{SIMULATION USING INITIAL DATA FROM HIGH CURRENT EXPERIMENT}

Encouraged by the above results, we synthesized an initial particle distribution for the HCX using 2-plane initially, and then 3-plane data. Our original attempt at folding in spatial density information employed time-integrated data from kapton film; we soon discovered that the timeintegrated distribution is significantly "smeared" due to variations in the beam properties over the pulse duration. Here we show results based on a single time-slice near mid-pulse, using crossed-slit measurements. The verticalmoving slit was downstream, so to obtain a consistent density at the position of the forward slit, the $y$ coordinate was "rescaled" using the mean envelope expansion derived from the parallel-slit data. These data are shown in Fig. 4. Using the initial particle set so generated, we ran a WARP simulation through the $(2.22 \mathrm{~m}, 10$ quad $)$ transport line; see also [5]. In Fig. 5 we show the measured beam at the
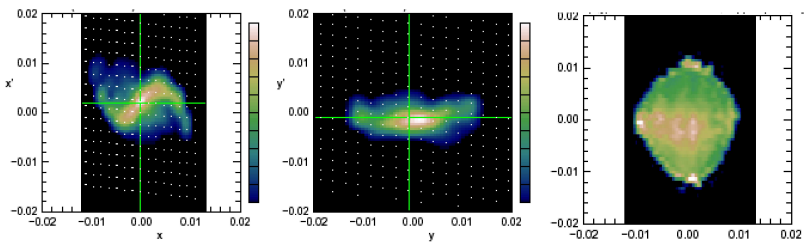

Figure 4: $\left(x, x^{\prime}\right),\left(y, y^{\prime}\right)$ and $(x, y)$ views of beam at upstream station QD1 of HCX electrostatic transport line. White dots in first two views denote actual data points.

downstream station "D-end" and the corresponding simulation results. While the beam dimensions and some important features such as the "hollowing" in the spatial density show rough agreement, and the absence of some features in the simulated beam can be explained by the fact that it was loaded in the center of the pipe while the experimental beam had shifted off-center, the agreement between the simulated and measured beams is far from ideal. We conjectured that correlations in unmeasured planes, $e . g$. $\left(y, x^{\prime}\right)$, were not being captured by the synthesis process. This gave increased impetus to the development of the new diagnostic capabilities described below and in [6].
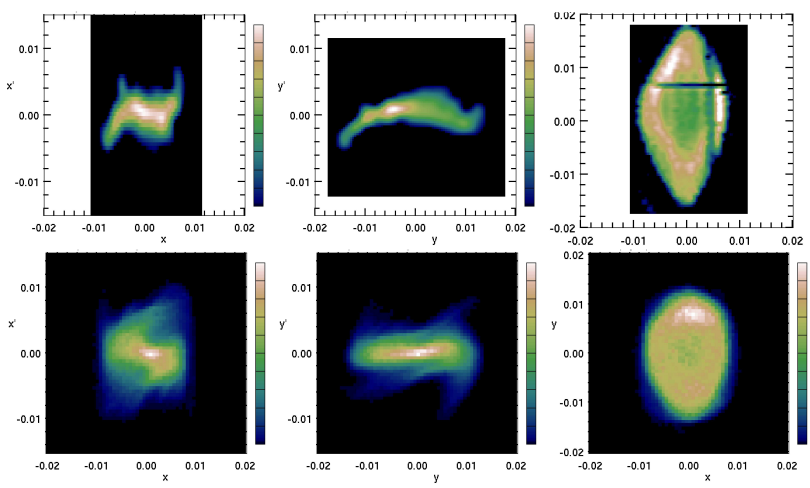

Figure 5: Top row: beam at downstream station D-end; bottom row: simulation result (same views).

\section{ANALYSIS OF 3D “OPTICAL SLIT” DATA}

We have started analyzing data from "optical slit" scans (two views, with slits moving in $x$ and $y$ respectively), recently carried out at the D-end station (2.22-m) of HCX. The apparatus is shown schematically in Fig. 6. We now describe the post-processing steps which are aimed at building up a self-consistent data set, and point out features of the derived data which support the view that nonzero correlations in the "other" planes are present.

For each pulse viewed using the horizontal scanner with the slit at some $x$, the raw image holds $f(u, v)$ at that $x$ : $x^{\prime}=(u-x) / d z_{h}$, so a pixel at $(x, u, v)$ contributes to $f\left(x, x^{\prime}, v\right)$ at $\left(x,(u-x) / d z_{h}, v\right)$. The beam distribution in the slit plane is a function of a "derived" coordinate $y$, which is presently rescaled from $v$ on the image plane using the mean beam envelope convergence in the vertical plane. 


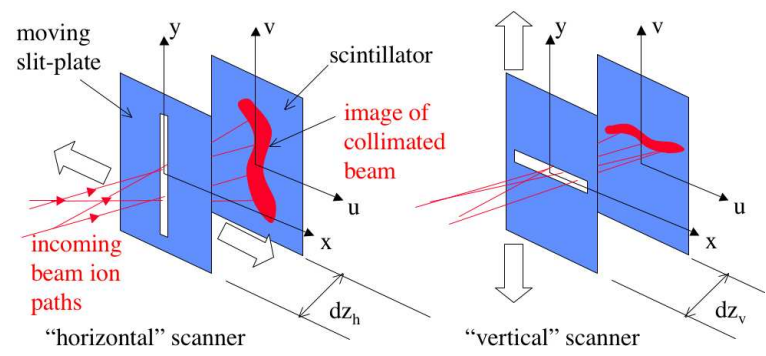

Figure 6: Schematics of "optical slit" scanners.

Thus, obtaining the $y$ values associated with each pixel for the horizontal data set requires use of the vertical data set. The mean convergence in the vertical plane, $\alpha_{y}$, is: $\alpha_{y}=$ $\left\langle(y-\bar{y})\left(y^{\prime}-\bar{y}^{\prime}\right)\right\rangle /\left\langle(y-\bar{y})^{2}\right\rangle$ (where the averages are $f$ weighted), and the transformation is: $y=v /\left(1+\alpha_{y} d z_{h}\right)$. The vertical scanner is handled similarly.

Fig. 7 contains views of the measured $f\left(x, y, x^{\prime}\right)$. In the first panel $f$ is averaged over $y$, as in a parallel-slit scanner. However, the latter two panels, showing $f\left(x,-1.95 \mathrm{~cm}, x^{\prime}\right)$ and $f\left(x, 0.8 \mathrm{~mm}, x^{\prime}\right)$, show that $f\left(x, y, x^{\prime}\right)$ is not independent of $y$. Note that this is the data set of [6] (Figs. 3-5 therein), and was taken using machine parameters differing from those of Figs. 4 and 5 of this paper.
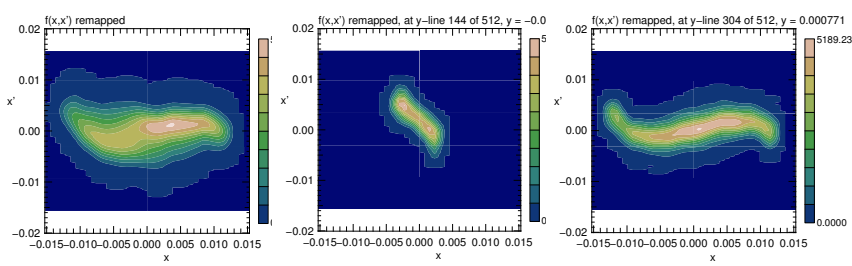

Figure 7: $\left(x, x^{\prime}\right)$ phase space from optical slit at D-end: integrated over $y$, and at two particular $y$ locations.
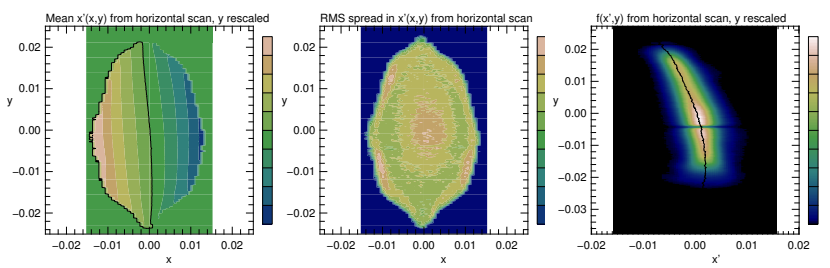

Figure 8: Optical slit data at D-end (see text).

The first two panels of Fig. 8 show moments of the distribution as functions of spatial position (at the slit plane): $\bar{x}^{\prime}(x, y)$ and $x_{r m s}^{\prime}(x, y)$. Note that $\overline{x^{\prime}}(x, y)$ is not truly linear in $x$ and independent of $y$ as it would be in a perfectly aligned system with no anharmonic forces. Note also the roughly factor-of-two variation in the transverse $(x)$ thermal speed evident in the second panel. The third panel shows $f\left(x^{\prime}, y\right)$ from the horizontal scan; note the dependence of the mean $x^{\prime}$ upon $y$, shown as a black trace running from top to bottom of the plot. (The horizontal line is the image of a support in the diagnostic). From this last view it is evident that extreme values of $x^{\prime}$ are found at the extremes in $y$ of the distribution. This feature contradicts the ansatz we had made in our syntheses, which employed a sampling region that disallowed exteme values of $x^{\prime}$ when $y$ was at its extremes; this may explain some of the descrepancy between simulation and experiment.

\section{PLANS}

We plan to improve the remapping of the raw data from the scintillator plane to the slit plane. As a first step, we will employ an $x$-dependent mean vertical slope, $\bar{y}^{\prime}(x)$, and a $y$-dependent mean horizontal slope, $\bar{x}^{\prime}(y)$. As can be seen in the last panel of Fig. 8, $\bar{x}^{\prime}$ is indeed not independent of $y$, and we expect that accounting for this will correct a distortion in the slit-plane spatial image obtained from the vertical scan. In general, we plan to retain centroid displacements and mean slopes, all of which are currently removed in the analysis, in future syntheses of $f$; this will require good fiducials in the experimental data. It may also be possible to use a spatially-dependent $\alpha$ to account for deviations from linear convergence or divergence.

The next step in our development of a benchmarked "local" simulation capability (as contrasted with a "source-toend" capability, which is also being pursued) will be to employ projectional 3D data (two or more views) or 4D data (sampled and interpolated) as the basis of the distribution synthesis. It is planned that a compact version of the optical slit diagnostic will be fielded at the upstream QD1 station of HCX, and when that data is available we will carry out the synthesis and simulations.

To synthesize a distribution, we may use the optical slit data to specify spatial bin counts $N(x, y)$ and probability distributions $f\left(x^{\prime}\right)$ and $f\left(y^{\prime}\right)$ at each cell in $(x, y)$, and load the particles in that cell with random $x^{\prime}$ values obeying those distributions. It would also be possible to employ a sampling region that excludes the "corners" in $\left(x^{\prime}, y^{\prime}\right)$ for particles in a spatial cell. Correlations in that plane are not measured by the optical slits, but may in the future be inferred from hole-plate data.

Multi-hole plates directly yield 4D data, but that data is sparse. It is hoped that interpolations in the velocity distribution, combined with a direct measurement of the spatial density, will yield accurate estimates of $f\left(x, y, x^{\prime}, y^{\prime}\right)$.

\section{REFERENCES}

[1] Seidl, P. A., et. al., these Proceedings.

[2] Friedman, A., Grote, D. P., Celata, C. M., and Staples, J. W., "Use of projectional phase space data to infer a 4D particle distribution," Laser and Particle Beams, in press.

[3] Grote, D. P., Friedman, A., Craig, G. D., Haber, I., and Sharp, W. M., Nucl. Instr. and Meth. A. 464, 563 (2001).

[4] Celata, C. M., Friedman, A., Grote, D. P., Haber, I., Henestroza, E., et. al., "Particle-in-Cell Simulations of Beam Dynamics in the HCX," Laser and Particle Beams, in press.

[5] Celata, C. M., et. al., these Proceedings.

[6] Bieniosek, F. M., et. al., these Proceedings. 
University of California

Lawrence Livermore National Laboratory

Technical Information Department

Livermore, CA 94551

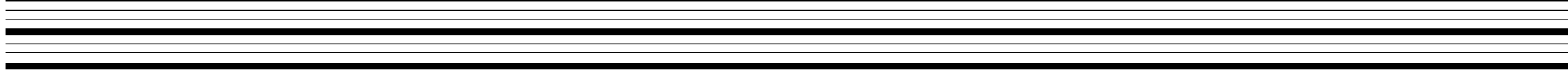

